\title{
ON THE EFFECT OF BINARY ENCOUNTERS
}

Tsutomu Shimizu

Bukkyo University, Kyoto 603, Japan

\section{INTRODUCTION}

The effect of binary encounters is examined by restricting considerations to a sphere of finite radius $R$, instead of an infinite one assumed hitherto. The sphere is centered at an assigned test-particle with velocity $\nabla_{0}$. The corresponding modified assumption is that particles lying outside the sphere are scattered randomly with an average number density $\mathrm{n}$ and their velocity distribution is Maxwellian with an r.m.s. of $\sqrt{3} \sigma$ in the space velocity. As for the particles' masses the same $M$ is presumed. Now the number of particles, with relative velocities $\nabla(V, \theta, \varphi)$ referred to the test-one, entering into a spherical band between the colatitudial angles $\chi$ and $\chi+d \chi$ per unit time, $d N_{1}$, is given by multiplying $n V \cos \theta \cdot 2 \pi R^{2} x$ $\sin x \mathrm{~d} x$ with the frequency distribution of $\nabla$ as follows (c.f. Fig. 1 ).

$$
\begin{aligned}
d N_{1}=2 \sqrt{\frac{2}{\pi}} n R^{2} \sigma \operatorname{Exp}\left[-Y_{0}^{2}-Y^{2}+2 Y Y Y\right. & (\cos \theta \cos x+\sin \theta \sin x \cos \varphi)] Y^{3} d Y \\
& \times \sin \theta \cos \theta d \theta d \varphi \sin x d x, \quad(0 \leq \theta \leq \pi / 2),
\end{aligned}
$$

where $\mathrm{Y}=\mathrm{V} /(\sqrt{2} \sigma)$ denotes the non-dimensional relative speed of a fieldparticle and $Y_{0}=V_{0} /(\sqrt{2} \sigma)$ the non-dimensional speed of the test-one.

\section{DENSITY DISTRIBUTION OF ENCOUNTING PARTICLES}

Taking into account that the encounter phenomena are symmetric around


Fig. 1. Notations concerning an encounting particle's orbit. 
the z-axis (c.f. Fig. 1) let us evaluate the number density per unit time of the entered particles at a point with radius $r$ and colatitudial angle $x^{\prime}, \nu\left(r, x^{\prime}\right)$. Since each of the entered particles moves supposedly along a Keplerian orbit, its position and its motion at some instant are expressed in terms of $Y, Y_{0}, \theta, \varphi$, and $\chi$. Hence, the change of variables from $X$ and $\varphi$ to $X^{\prime}$ and $\varphi^{\prime}$ in eq. (1) provides information on the number of encounting particles in a volume element $2 \pi r^{2} d r \sin x^{\prime} d x^{\prime}$. However, the timeinterval for staying in a spherical shell between the radii $r$ and $r+d r$, namely $\mathrm{dr} /|\dot{\mathrm{r}}|$ ( $\dot{\mathrm{r}}$ : the radial velocity at $r$ ) depends on $r$, so that it must be taken into account. Then, letting $X$ be $r / R$ and since $\sin x d x d \varphi=\sin x^{\prime} d x^{\prime} d \varphi^{\prime}$ we have

$$
\begin{aligned}
\nu\left(X, x^{\prime}\right) 2 \pi r^{2} d r \sin X^{\prime} d X^{\prime} & =2 \sqrt{\frac{2}{\pi}} n R^{2} \sigma \int_{\alpha_{1}}^{\infty} \operatorname{Exp}\left(-Y_{0}^{2}-Y^{2}\right) Y^{3} d Y \int_{0}^{\theta_{1}} \frac{d r}{|\dot{r}|} \sin \theta \cos \theta d \theta \\
& \times \int_{0}^{2 \pi} \operatorname{Exp}\left[2 Y_{0} Y(\cos \theta \cos x+\sin \theta \sin x \cos \varphi)\right]\left(\sin x^{\prime} d x^{\prime}\right) d \varphi^{\prime},
\end{aligned}
$$

where the integral range of $\mathrm{Y}$ is taken in order to exclude the elliptic orbits, since the escape velocity at $R$ corresponds to $\sqrt{2 G M /\left(\sigma^{2} R\right)}=\alpha_{1}$, while that of $\theta$ is conditioned so as to reject the imaginary radial velocity by putting $\theta_{1}=\operatorname{Sin}^{-1} \sqrt{\alpha_{1}^{2} X(1-X) / Y^{2}+X^{2}}$. After cancelling drsinx $x^{\prime} x^{\prime}$ on both sides and carrying out some calculations eq. (2) is reduced to

$$
\begin{aligned}
\nu\left(X, X^{\prime}\right) & =\frac{2}{\sqrt{\pi}} \frac{n \alpha_{1}^{3}}{x^{2}} \int_{1}^{\infty} \operatorname{Exp}\left(-Y_{0}^{2}-\alpha_{1}^{2} y^{2}\right) y^{3} d y \int_{0}^{\theta_{1}} \frac{1}{\rho} \sin \theta \cos \theta d \theta \\
\times & \left\{\operatorname{Exp}\left[2 \alpha_{1} Y_{0} y \cos (W-w+\theta) \cos x^{\prime}\right] I_{0}\left[2 \alpha_{1} Y_{0} y \sin (W-w+\theta) \sin x^{\prime}\right]\right. \\
& \left.+\operatorname{Exp}\left[2 \alpha_{1} Y_{0} y \cos (W+w+\theta) \cos x^{\prime}\right] I_{0}\left[2 \alpha_{1} Y_{0} y \sin (W+w+\theta) \sin x^{\prime}\right]\right\},
\end{aligned}
$$

where $y$ denotes $Y / \alpha_{1}, \rho=|\dot{r}| /\left(\sqrt{2} \sigma \alpha_{1}\right)$ the non-dimensional radial velocity, $\mathrm{W}$ the true anomaly at an incident point $\mathrm{P}$ on the sphere, $\mathrm{W}$ the true
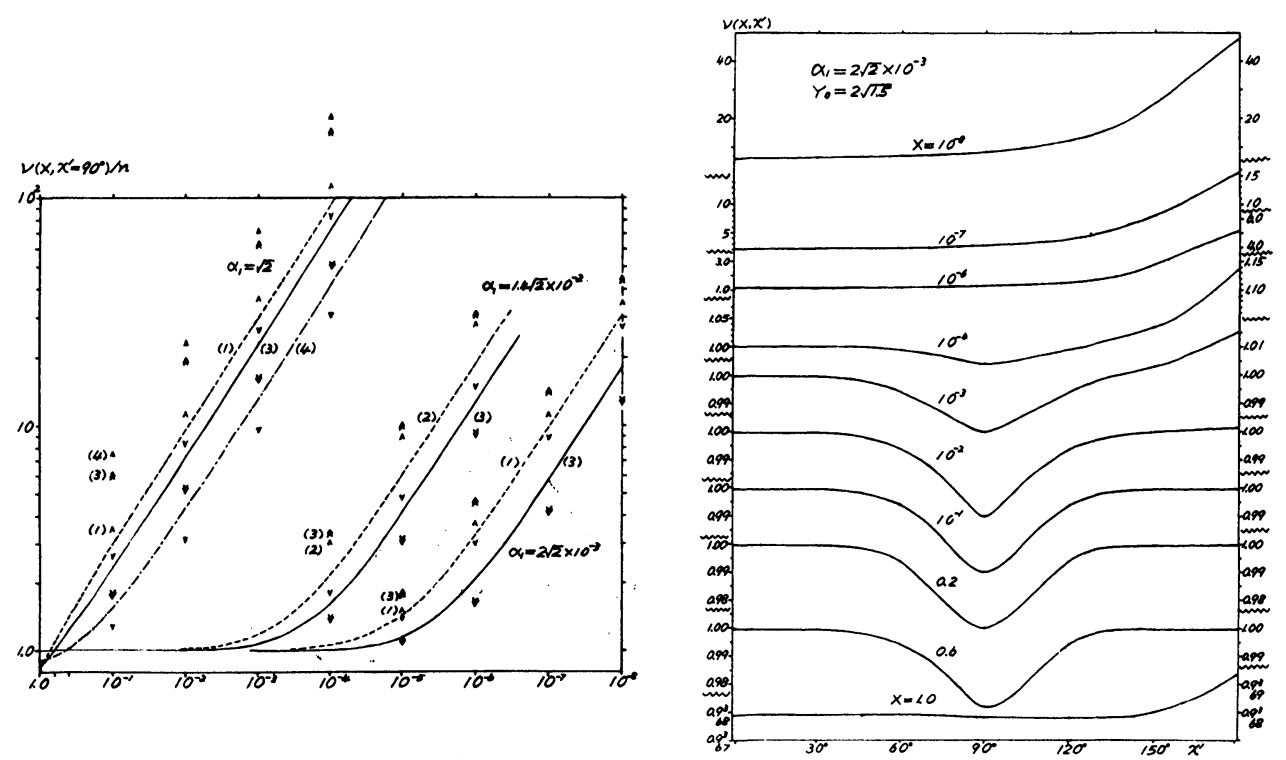

Fig. 2. Dependence of $\nu\left(X, x^{\prime}\right)$ on $X$. Fig. 3. Dependence of $\nu\left(X, x^{\prime}\right)$ on $x^{\prime}$. 
anomaly at $r$, and $I_{0}(x)=\sum_{i=0}^{\infty}(x / 2)^{2 i} /(i ! i !)$ the modified Bessel function of the zeroth order. It should be noticed that the second and the third line in eq. (3) correspond to the particles going into and those going out from a sphere with radius $r$ respectively.

From eq. (3) the number of particles per unit time in a spherical shell between the radii $r$ and $r+d r, d N(X)$, is derived as follows.

$$
d N(X)=4 \sqrt{\pi} n R^{3} \frac{\alpha_{1}^{2}}{Y_{0}} x^{3 / 2} d X \int_{1}^{\infty}\left\{\operatorname{Exp}\left[-\left(Y_{0}-\alpha, y\right)^{2}\right]-\operatorname{Exp}\left[-\left(Y_{0}+\alpha_{1} y\right)^{2}\right]\right\} \sqrt{1+X\left(y^{2}-1\right)} d y .
$$

This indicates that the proportionality ofdN $(X)$ to $X$ holds more closely as $X$ becomes smaller. Accordingly a nearly dependence of $\nu\left(X, X^{\prime}\right)$ on $X^{-1 / 2}$ is expected for sufficiently small values of $X$.

Our numerical results substantiate it as exemplified in Fig. 2, where full and broken lines indicate runs of $\nu\left(X, X^{\prime}=90^{\circ}\right) / n$, while $\nu\left(X, X^{\prime}=179^{\circ}\right) / n$ and $\nu\left(X, X^{\prime}=1^{\circ}\right) / \mathrm{n}$ are specified by upward marks $(\Delta, \wedge$, or $\hat{\wedge})$ and downward ones $(\nabla, \vee$, or $\Downarrow)$ respectively. In the vicinity of the test-particle, where such linearity holds approximately, the dependence of $\nu\left(X, \chi^{\prime}\right)$ on $\alpha_{1}$ appears to be roughly linear for a certain set of $Y_{0}$ and $\chi^{\prime}$.

As for the relationship between $\nu\left(X, X^{\prime}\right) / n$ and $\chi^{\prime}$ it is illustrated in Fig. in which a dence crowding of particles is detectable in the stern of the test-particle. Such a trend is magnified as $\boldsymbol{\alpha}_{\mathbf{1}}$ increases and $Y_{0}$ increases.

\section{DYNAMICAL FRICTION}

Now the gravitational force acting on a unit mass of the test-particle due to the encounting particles lying outside an assigned radius $r, F(X)$, can be obtained by multiplying $\left(\mathrm{GM} / r^{2}\right) \cos x^{\prime} \cdot 2 \pi r^{2} d r \sin x^{\prime} d x^{\prime}$ with eq. (3) and integrating with respect to $\chi^{\prime}$ and $r$. After lengthy calculations we have

$$
\begin{aligned}
& F(X)=-\sqrt{\pi} \frac{n \mu^{2}}{\sigma^{2} Y_{0}} \operatorname{Exp}\left(-Y_{0}^{2}\right) \int_{1}^{\infty} \operatorname{Exp}\left(-\alpha_{1}^{2} y^{2}\right)\left[\cosh \left(2 \alpha_{1} Y_{0} y\right)-\frac{\sinh \left(2 \alpha_{1} Y_{0} y\right)}{2 \alpha_{1} Y_{0} y}\right] \frac{y d y}{\left(y^{2}-1\right)^{2}}\left\{1-\frac{\left(2 y^{2}-1\right)^{2}}{2 y^{2}\left(y^{2}-1\right)} \ln \left(2 y^{2}-1\right)\right. \\
& \quad-\frac{1}{y} \sqrt{\frac{1+x\left(y^{2}-1\right)}{x}}+\frac{(1-x)^{2}}{2 x y^{2}}\left(y^{2}-1\right) \ln \left|\frac{\sqrt{1+x\left(y^{2}-1\right)}-y \sqrt{x}}{\sqrt{1+x\left(y^{2}-1\right)}+y \sqrt{x}}\right|-\frac{\left[y^{2}+x\left(y^{2}-1\right)\right]}{2 x y^{2}\left(y^{2}-1\right)} \ln \left|\frac{\sqrt{1+x\left(y^{2}-1\right)}-\sqrt{x}(y-1 / 4)}{\sqrt{1+x\left(y^{2}-1\right)}+\sqrt{x}(y-1 / y)}\right| .
\end{aligned}
$$

For $X=\varepsilon \ll l$ all the terms in the second line of eq. (5) reduce to $4(y-1 / y) \sqrt{\varepsilon}$. Thus eq. (5) without the second line represents $F(0)$, namely the force due to all the particles in the whole sphere of the radius $R$. This $F(0)$ is proved to be identical with the velocity change per unit time of the test-particle, $\Sigma \mathbf{\Delta} v_{i l}$, derived under the same assumption as made here, so that $\mathrm{F}(\mathrm{X})$ is nothing but a part of the so-called dynamical friction. Numerical integrations of eq. (5) for different values of $X$ furnish information concerning which part of the hypothetical sphere contributes significantly to the encounter effect. Fig. 4 illustrates the dependence of $F(X) / F(O)$ on $X$ and the value of $F(O)$ for each value of $Y$ in the case of $\alpha_{1}=2 \sqrt{2} \times 10^{-3}$. It is found therein that the encounter effect on a testparticle is mainly caused by its neighbouring particles crowding backwards and the other ones lying in the outer part of the sphere contribute a little even though their number is much larger. Our results show that as $\alpha_{1}$ increases and $Y$ decreases a smaller range of $\log X$ covers nearly the same curve of $F(X) / F(O)$ as shown in Fig. 4. 




Fig. 4. $F(0)$ and dependence of $F(X) / F(O)$ on $X$.

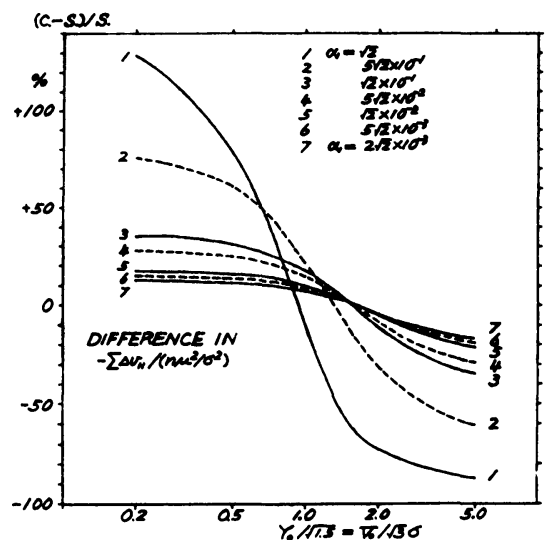

Fig. 5. Percentage difference in $\sum \Delta v_{11}((C-S) / S)$.

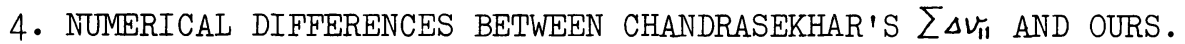

Here we compare numerical values from Chandrasekhar's formula of $\sum \Delta v_{11}$ with those from our $F(O)$. Supposing $D:=R$ and denoting $\Phi(x)$ the error integral, we can write the former in our notations as

$$
\sum \Delta v_{11}=-\pi \frac{n \mu^{2}}{\sigma^{2} Y_{0}^{2}} \ln \left(\frac{3}{\alpha_{1}^{2}}\right)\left[\Phi\left(Y_{0}\right)-Y_{0} \Phi^{\prime}\left(Y_{0}\right)\right] .
$$

Numerical differences in $\sum \Delta v_{11} /\left(n \mu^{2} / \sigma^{2}\right)$ between eq. (6) and $F(0)$ from eq. (5) are shown graphically in Fig. 5 with different values of $\alpha_{1}$ and $Y$. It is found there that deviations remain insignificant so long as $\alpha_{1}$ is small enough as for the stellar field in the solar neighbourhood, but for a larger $\alpha_{1}$ as in the cores of globular clusters and of clusters of galaxies the deviation becomes larger as $Y_{0}$ departs far from $\sqrt{1.5}$ corresponding to $V_{0}=\sqrt{3} \sigma$. This may be anticipated since the initial assumption in deriving eq. (6) was $\mathrm{R} \rightarrow \infty$, so $\alpha_{1} \rightarrow 0$. On this connection it should be remarked that the impact parameter and the inclination of the orbital plane have usually been taken as the pair of independent variables, while in our treatment the colatitudial angle and the azimuthal one of the incident point on the sphere are adopted, consequently a modification sets in even when $R$ tends to infinity.

\section{Reference.}

Chandrasekhar, S.: 1943, "Astrophys. J." 97, pp. 255-273. 